\title{
Recurrent Laryngeal Nerve Identification During Thyroidectomy
}

\author{
Yasser M. Hatata MD FRCS, Ghada Morshed MD MRCS.
}

\author{
Departments of Surgery, Faculty of Medicine Fayoum University
}

Objectives: To assess the applicability of intraoperative recurrent laryngeal nerve (RLN) identification during thyroidectomy. Recurrent laryngeal nerve injury is one of the most serious complications of surgery in the neck. Numerous studies have demonstrated the utility of intraoperative monitoring of the RLN in adult thyroid surgery to prevent such injury.

Patient and methods: It is a prospective study which included 50 patients undergoing thyroidectomy. During surgical excision, intraoperative RLN identification was performed with use of methylene blue. True vocal fold mobility was examined by postoperative flexible laryngoscopy.

Results: Intraoperative RLN identification was performed successfully for all patients and we avoided postoperative RLN paresis in all cases.

Conclusion: Intraoperative RLN identification with methylene blue can be a useful tool during thyroidectomy and it is a safe and reliable technique.

Key words: Thyroidectomy, recurrent laryngeal nerve, methylene blue.

\section{Introduction:}

Thyroid surgery is one of the commonly performed surgery these days. ${ }^{1}$ Recurrent laryngeal nerves are closely related to the inferior thyroid artery. Injuries involving recurrent laryngeal nerves can cause morbidities ranging from aspiration to stridor. The best way to avoid injuries to recurrent laryngeal nerve is to identify the nerve in all cases. ${ }^{2,3}$

Thyroidectomy for large cervical or substernal goiters, thyroidectomy for Grave disease, surgery after radiation therapy or radioactive iodine treatment, and in cases where bilateral paratracheal dissection or bilateral thyroidectomy, injury to both RLNs is possible. ${ }^{4}$ Based on the demonstrated safety, we use methylene blue during surgery for identification of recurrent laryngeal nerve.

The aim of this study is to describe a new and simple technique for recurrent laryngeal nerve identification.

\section{Patients and methods:}

This is a prospective study which included 50 patients undergoing thyroidectomy, the study was undertaken in El Fayoum University Hospital from July 2013 till June 2014. There were 33 females aged between 35 and 50 years and 7 males aged between 40 and 55 (mean age $41.7 \pm 12.6$ ). The indirect laryngoscopy (IDL) was performed by the ear, nose and throat (ENT) registrar the day before surgery.

We included patients with simple multinodular goiter, secondry toxic goiter, and solitary thyroid nodule and thyroid neoplasms. We took a written informed consent from all patients, we excluded patients with coagulopathy, international normalized ratio (INR) below 1.4, patients with previous neck surgery, patients with preoperative voice symptoms or preoperative abnormalities to the vocal cords during ENT examination and patients who were unable to understand the study purpose.

General anesthesia was used. During surgical excision, intraoperative RLN identification was performed with use of methylene blue by spraying the thyroid tissue. All the thyroid tissue stained blue with methylene blue except the recurrent laryngeal 


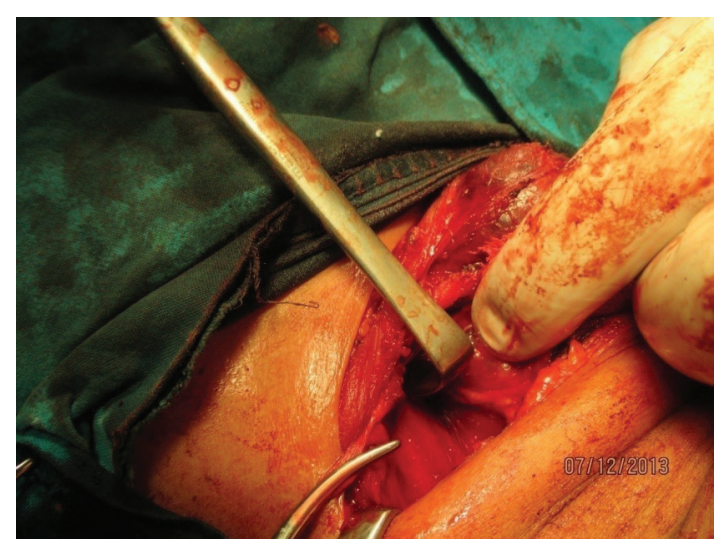

Figure (1): Recurrent laryngeal nerve before spraying methylene blue.
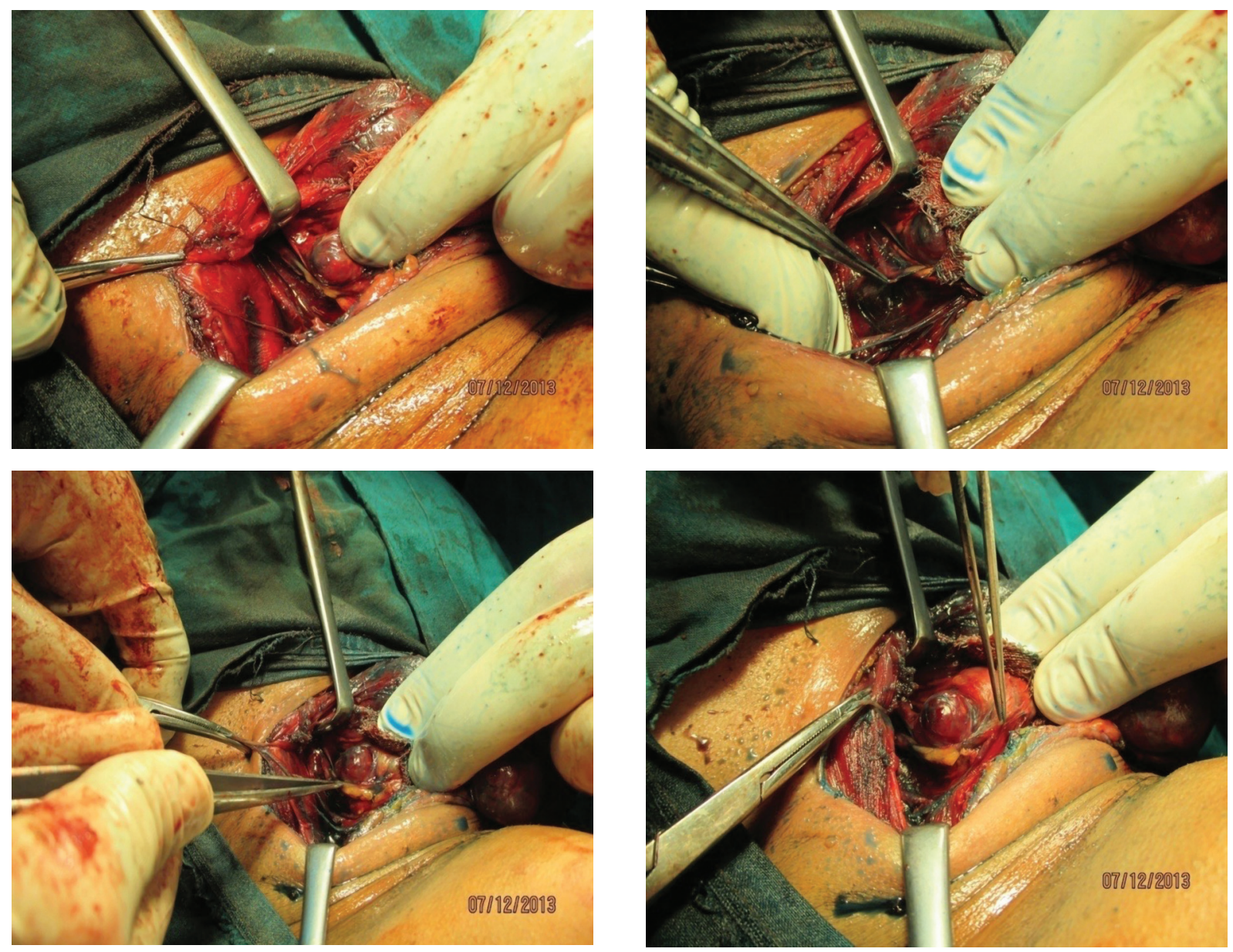

Figures (2-5): Recurrent laryngeal nerve after spraying methylene blue.

nerve that did not take the dye, the parathyroid glands kept stained for 2 minutes then they regained their colours after that Figures (1-5). True vocal fold mobility was examined by postoperative flexible laryngoscopy.

\section{Results:}

The study included 50 patients $33(66 \%)$ females and $17(34 \%)$ males. The mean age at diagnosis was $41.7 \pm 12.6$ (range 35-55years), the mean duration for the procedures was 95.3 \pm 17.4 (range $85 \mathrm{~min}-112 \mathrm{~min}$ ). A total of 10 thyroidectomies, 33hemithyroidectomies and 7 subtotal thyroidectomies were performed.

Intraoperative RLN identification was performed successfully for all patients and we avoided postoperative RLN paresis in all cases. There were no complications nor 
mortalities and the mean hospital stay was 2days (range1-3days).

\section{Discussion:}

The previous controversy regarding routine RLN identification during thyroidectomy has been resolved in favor of routine identification and dissection in all cases. 5,6

Variations in the anatomic position of the RLN can occur and the risk of nerve injury increases in patients with anomalous anatomy of the RLN. Such anomalies include RLN displacement by thyroid nodularity or paratracheal lymphadenopathy, nonrecurrence of the RLN, extralaryngeal branching of the RLN, and variations of the course of the nerve in relation to the inferior thyroid artery and ligament of Berry. In a recent study of RLN anatomy in 491 thyroid surgery cases, $60.8 \%$ of RLNs were found in the expected tracheoesophageal groove position, whereas $4.9 \%$ were lateral and $28.3 \%$ were posterior to the trachea; of greatest concern are those cases where the RLN was found on the anterior surface of the thyroid gland, a particularly high-risk area for nerve injury. ${ }^{7}$ In our study in 48 cases the RLN was at the tracheoesophageal groove and in 2 cases was found posterior to the trachea.

In our study it is well established that visual identification of the RLN during thyroid surgery reduces the incidence of iatrogenic RLN palsy as in other studies. 5,8

\section{Conclusion :}

Intraoperative RLN identification with methylene blue can be a useful tool during thyroidectomy and it is a safe and reliable technique.

\section{Reference:}

1- Al-Sobhi SS: The current pattern of thyroid surgery in Saudi Arabia and how to improve it. Ann Saudi Med 2002; 22(3-4): 256-257.

2- Sosa JA, Bowman HM, Tielsch JM, Powe NR, Gordon TA, Udelsman R: The importance of surgeon experience for clinical and economic outcomes from thyroidectomy. Ann Surg 1998; 228 (3): 320-330.

3- Lamadé W, Renz K, Willeke F, Klar E, Herfarth C: Effect of training on the incidence of nerve damage in thyroid surgery. Br J Surg 1999; 86 (3): 388-391.

4- Randolph GW: Surgical anatomy of the recurrent laryngeal nerve. Randolph GW surgery of the thyroid and parathyroid glands. Philadelphia, PA Elsevier Science 2002; 300-342.

5- Randolph GW: Surgical anatomy of the recurrent laryngeal nerve. Randolph GW surgery of the thyroid and parathyroid glands. Philadelphia, PA Elsevier Science 2002; 300-342.

6- Gavilán J, Gavilán C: Recurrent laryngeal nerve: Identification during thyroid and parathyroid surgery. Arch Otolaryngol Head Neck Surg 1986; 112 (12): 1286-1288 PubMed.

7- Hisham AN, Lukman MR: Recurrent laryngeal nerve in thyroid surgery: A critical appraisal. ANZ J Surg 2002; 72 (12): 887889 PubMed.

8- Marcus B, Edwards B, Yoo S, et al: Recurrent laryngeal nerve monitoring in thyroid and parathyroid surgery: The University of Michigan experience. Laryngoscope 2003; 113 (2): 356-361 PubMed. 


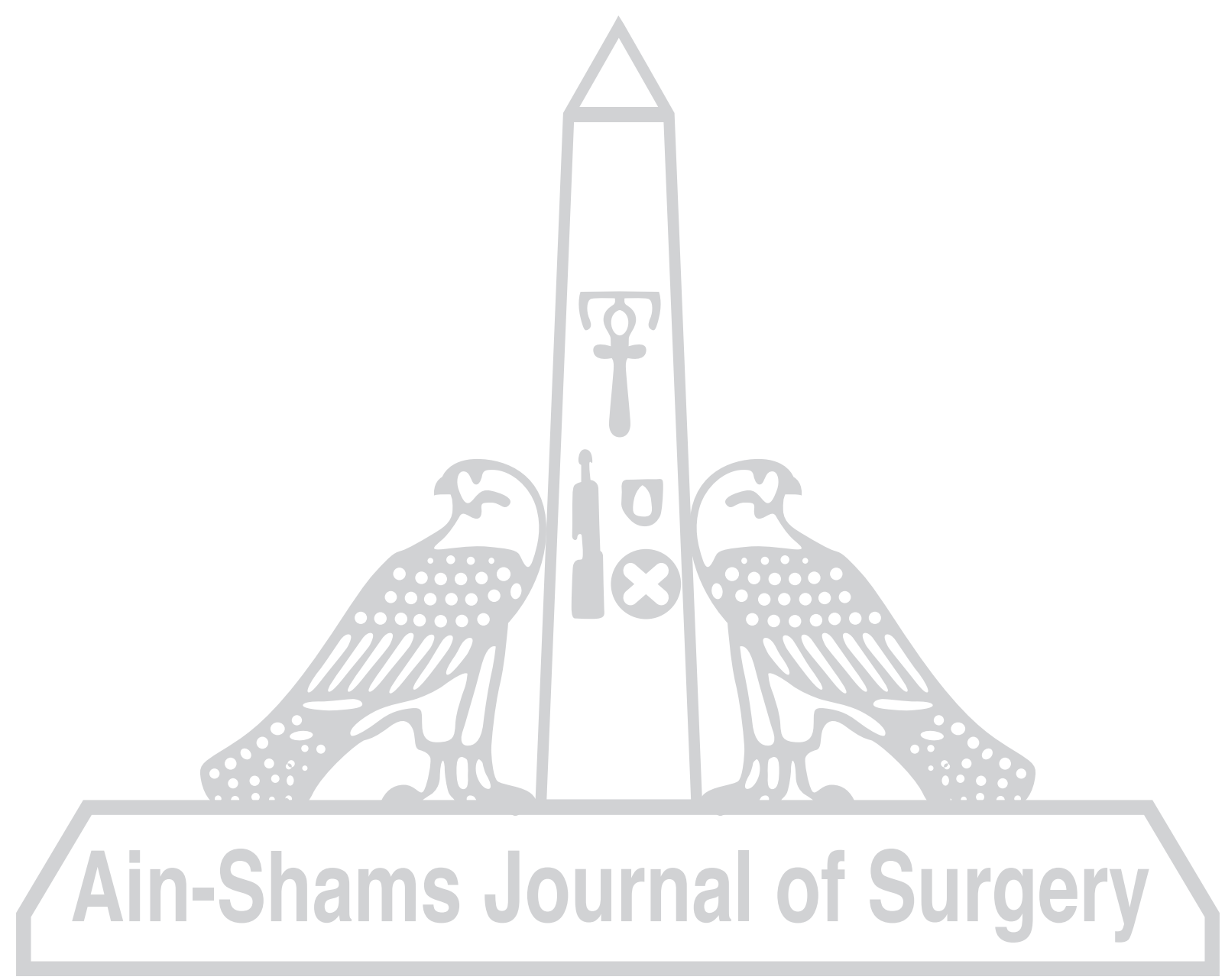

